\title{
Revista de la
}

\section{CEPAL}

Secretario Ejecutivo

Gert Rosenthal

Secretario Ejecutivo Adjunto

Andrés Bianchi

\section{Director de la Rerista}

Anibal Pinto

Secretario Técrico

Eugenio Lahera

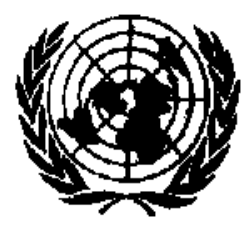

NACIONES UNIDAS

COMISION ECONOMICA PARA AMERICA LATINA Y EL CARIBE

SANTIAGO DE CHILE, DICIEMBRE 1988 


\section{Revista de la}

\section{CEPAL}

\section{SUMARIO}

Competitividad internacional: evolución y lecciones. F. Fajnzylber

Revolución industrial y alternativas regionales. H. Nochteff

Cambio técnico y reestructuración productiva. $E$. Lahera

Notas sobre la automatización microelectrónica en el Brasil. J.R. Tauile

Exportaciones e industrialización en la Argentina, 1973-1986. D. Azprazu y B. Kosacoff

Politica social rural en una estrategia de desarrollo sostenido. $J$. Durston

Interacción de los sectores público y privado y la eficiencia global de la economía. J.M.F. Martin

El problema de la deuda de Cuba en monedas convertibles. A.R.M. Ritter

La seguridad alimentaria; tendencias e impacto de la crisis. A. Schejtman

Economias de viabilidad difícil; una opción por examinar. A. Núñez del Prado

La génesis de la sustitución de importaciones en América Latina. R.L. Ground 


\section{REVISTA DE LA CEPAL, $\mathbf{N}^{\circ}$ 36}

\section{Competitividad internacional: evolución y lecciones}

\author{
Fernando Fajnzylber*
}

El tema de la reestructuración productiva e incorporación de progreso técnico constituye una preocupación de las naciones industrializadas y de los países en desarrollo, sea que en sus economías predomine el mercado o la planificación.

La perspectiva con que encaran este proceso las naciones industrializadas difiere radicalmente de la que prevalece en América Latina. En las primeras, la reestructuración productiva persigue el mejoramiento de la competitividad, entendida como la capacidad de un pais para exponerse al mercado externo y mantenet o elevar el nivel de vida de su población. En América Latina, en cambio, el propósito básicu apunta, con algunas excepciones recientes, a generar un superávit comercial suficiente para servir la cuantiosa deuda externa, lo cual no necesariamente se traduce en avances en materia de competitividad y exige a menudo reducir el precario nivel de vida de amplios segmentos de la poblacion. Tal es la diferencia entre competitividad con incorporación de progreso técnico, que es la que procuran las naciones indusurializadas, y aquella que se funda en la contracción del ingreso.

Existe una amplia gama de interpretaciones res. pecto a los factores que habrian desencadenado este proceso en las naciones industrializadas, asi como a sus consecuencias en los ámbitos económico, social, polftico y cultural. A esta extensa gama de interpretaciones se asocia una variedad semejante de recomendaciones de política.

En este artículo el autor intenta caracterizar esquemáticamente la situación imperante en los palses avanzados. Para tal efecto, examina los diferentes factores que explican este proceso, poniendo énfasis en el tema de la competitividad internacional. Destaca, asimismo, la experiencia de algunos de esos paises, que puede resultar útíl para el debate regional.

*Oficial a cargo de ta División Conjunta cepatonunt de Desarrollo Indusirial y Tecnologia, Saniago de Chile.

\section{I \\ Factores explicativos de la reestructuración productiva}

Las diversas interpretaciones formuladas en la última década en torno a este proceso difieren en la identificación de sus detonantes principales, en las vinculaciones entre éstos y en la forma en que gravitan sobre la realidad.

Algunos de los factores que difícilmente pueden ser ignorados en una interpretación que pretenda cubrir la complejidad del tema de la reestructuración productiva son: i) alza espectacular en el pasado decenio y errática trayectoria posterior del precio del petróleo; ii) desorden financiero y aumento explosivo de la liquidez; iii) saturación del patrón de consumo imperante desde mediados de siglo; iv) transición hacia un nuevo patrón tecnológico, cuyo eje son "las tecnologías de información" ( $\mathrm{Tl})$; v) espectacular mejoramiento de la competitividad comercial de Japón y de los nuevos países industrializados (NIC's) del Asia respecto del resto del mundo; $y$ vi) agravamiento de los desequilibrios fiscal y externo de los Estados Unidos, pivote y referente básico de la expansión económica mundial en la postguerra.

Aunque de variada índole, estos factores se encuentran vinculados entre $s 1$, y en el transcurso de los últimos años han venido siendo objeto de cambiantes prioridades. En el período posterior a 1973 la atención se centró en la estampida del precio del petróleo y la expansión desmesurada de la liquidez, factores que se vinculan por el reciclaje de los ingresos captados por las naciones productoras de crudo. Hacia fines de los años setenta, cuando se toma conciencia de los orígenes estructurales de la pérdida de dinamismo de la economía mundial, se privilegian los demás factores. Estos últimos se encuentran interrelacionados, en la medida que los protagonistas -Japón y los Nic's asiáticos- han logrado éxito en el empeño de incorporar en nuevos bienes de consumo transables internacionalmente así como en los equipos y procesos requeridos para su producción, las tr gestadas y desarrolladas a nivel de ingenieria en actividades asociadas principalmente al programa espacial y militar de los Estados Unidos.

Al promediar la década en curso los desequilibrios de la economía norteamericana alcanzan 
magnitudes sin precedentes. Ello pone de manifiesto la precariedad del crecimiento que ese país había exhibido desde 1983 y que había servido de locomotora de la economía mundial.

Varias son las hipotesis entre las que se puede optar en lo tocante a las relaciones de causalidad que se dan entre los factores mencionados. Sin embargo, es innegable que todos ellos han de ser considerados cuando se trate de formular políticas en el ámbito de la reestructuración productiva. Cabe reconocer, empero, que en los palses industrializados el tema de la competitividad domina el debate académico y político y en torno a él se organiza el resto del temario. En estos paises la reestructuración productiva se entiende básicamente como la necesidad de adaptación frente al desafio de la competitividad.

La escuela de pensamiento que centra su análisis en el cambio tecnologico ${ }^{1}$ sostiene que la superación del actual ciclo, caracterizado por escaso dinamismo global, supone la introducción en los ámbitos económico, social y político, de innovaciones que permitan absorber $y$ potenciar en plenitud el nuevo "paradigma técnico econó mico". Algunas de sus principales secuelas serían: i) pérdida de significación de las economías de escala basadas en la producción masiva con técnicas de uso intensivo de capital; ii) mayor integración dentro de la empresa de las funciones de diseño, producción, adquisiciones e investigación y desarrollo; iii) capacidad para modificar productos y procesos en breve plazo; articulación de redes integradas de proveedores de partes y componentes, plantas de ensamblaje, distribuidores y laboratorios de investigación y desarrollo, con importante ahorro de capital; $y$ v) surgimiento de nuevas actividades de servicios vinculadas a la producción (software, diseño, información técnica), las que podrán ser desempenaadas por empresas de tamaño reducido.

Esta línea argumental requiere algunas precisiones. Sin duda, el debate sobre la vigencia del "Estado de bienestar" en las naciones industrializadas $\rightarrow$ y en alguna medida, en las de economía planificada-obedece a la "amenaza" que repre-

${ }^{1}$ Veanse Schumpeter (1950), Mensch (1979), Freeman y Soete (1982, 1985), Freeman (1987). Un cuestionamiento a fondo acerca de la vigencia de las "ondas largas" aparece en Rosenberg y Frischtak (1984), pp. 7-24. senta el incremento de la competitividad internacional del Japón y sus seguidores asiáticos. Las TI requieren, $y$ hacen viables, modificaciones institucionales, pero se percibe con nitidez el peligro que entraña la creciente incapacidad para competir en los mercados internacionales.

Ahora bien, la sorprendente arremetida japonesa en la economía mundial -y muy especialmente en el mercado estadounidense- se explica tanto por factores externos como domésticos. Entre los primeros, el más determinante es la magnitud, dinamismo $y$ apertura de la economía norteamericana pese a las variadas presiones proteccionistas que se han ejercido a nivel sectorial. Entre los factores domésticos, que explicarían la excepcional eficacia y rapidez con que el Japón habría incorporado las Ti en productos y procesos, descuellan: i) la capacidad, a nivel nacional y empresarial, para identificar áreas tecnológicas de importancia estratégica a mediano y largo plazo; ii) la existencia de mecanismos institucionales capaces de canalizar hacia esas áreas cuantiosos recursos para la inversión y el desarrollo tecnológico; iii) la flexibilidad de la estructura industrial, apoyada en la particular vinculación entre conglomerados líderes y la pequeña y mediana industria; y iv) la aproximación sistémica al diseño de productos y procesos y la integración de labores de concepción y fabricación.

Es innegable, también, que el peculiar sistema financiero internacional ejerce - aparte de una influencia directa en la economía real- un gran impacto institucional ya que incluso llega a hacer dudar de la autonomía de los Estados nacionales para definir sus politicas económicas.

Cabría argumentar que tanto en el caso del Japon como en el funcionamiento del sistema financiero internacional, las $\mathrm{TI}$ desempeñan un papel primordial, pero ello no debe ser obstáculo para reconocer la trascendencia y el perfil propio de cada uno de esos fenómenos. Por su parte, la evolución posterior de la economia norteamericana y su relación de competitividad con Japón y Alemania Federal ejercerán gran influencia en los demás factores. Las soluciones "institucionales" que adopte ese país condicionarán en alguna medida el desempeño del sistema financiero, la naturaleza de la reestructuración que deben emprender Japón y Alemania Federal, la intensidad y modalidades de masificación en el uso de las $\mathrm{TI}$, la gestación a la larga de un 
nuevo patrón de consumo y, en menor grado, la evolución del mercado petrolero.

A continuación se compara de manera es- quemática la inserción internacional de esos tres paises, con el objeto de configurar un marco de referencia para el análisis posterior.

\section{II \\ La inserción internacional de los países industriales líderes}

Los tres países considerados -Estados Unidos, Japón y Alemania Federal- tienen una población que representa alrededor del $9 \%$ de la población mundial y es semejante a la de América Latina. Sin embargo, su gravitación economica es impresionante, ya que generan el $40 \%$ del producto mundial, y su productividad cuadruplica el promedio mundial. Por otra parte, concentran, respectivamente, casi la mitad y alrededor del $75 \%$ de los recursos que la comunidad internacional y la ocDE destinan a investigación y desarrollo; el gasto por habitante que efectúan en estos rubros quintuplica el promedio mundial.

Por las razones expuestas, el comportamiento de estos países configura el perfil del sistema industrial mundial y es demostrativo de sus rasgos principales. Más allá de las actuales tensiones de tipo comercial, es incuestionable que el tipo de producto, procesos y modalidades de fabricación que prevalecen en la economfa mundial son el fruto de la interacción de los tres países, lo mismo que los acuerdos institucionales y la accesibilidad que las demás naciones puedan tener a la evolución futura del conocimiento en los distintos sectores industriales.

Entre los Estados Unidos, de un lado, y Japón y Alemania Federal, del otro, hay algunas diferencias importantes (cuadro 1). El primero, posee una base de producción científica mucho más amplia. Su densidad de autores cientificos con relación a su población septuplica el promedio mundial, en tanto que Alemania Federal lo cuadruplica y el Japón sólo lo dobla. En abierto contraste, la producción manufacturera de estos dos países considerados en conjunto, es ya casi $20 \%$ superior a la estadounidense, pese a que su población es un quinto menor. El desequilibrio que acusa Estados Unidos parece atribuible en
Cuadro 1

GRAVITACION ECONOMICO-TECNOLOGICA DE ESTADOS UNIDOS, JAPON

Y ALEMANIA FEDERAL HACIA 1980

(Porcentaje respecto del total mundial)

\begin{tabular}{lrrc}
\hline & $\begin{array}{r}\text { Estados } \\
\text { Unidos }\end{array}$ & Japón & $\begin{array}{c}\text { Alemania } \\
\text { Federal }\end{array}$ \\
\hline 1. Población & $\mathbf{5 . 0}$ & 2.5 & $\mathbf{1 . 3}$ \\
2. Producto interno bruto & 27.0 & 9.4 & 5.8 \\
3. Producto manufacturero & 18.0 & 11.7 & 9.4 \\
4. Bienes de capital & 14.7 & 11.1 & $\mathbf{9 . 6}$ \\
5. Ingenieros y científicos & 17.4 & 12.8 & $\mathbf{3 . 4}$ \\
6. Recursos en investigación & & & \\
y desarrollo tecnológico & 30.1 & 10.2 & 6.7 \\
7. Autores cientificos & 95.0 & 4.9 & 5.4 \\
\hline
\end{tabular}

Fuente: División Conjunta CEPaL/ONUd, sobre la base de información de Naciones Unidas, ONUD1, UNRSCo. Internatio. nal Science and Techrology Dota, Updated 1986, National Science Foundation and Current Bibliographical Directory.

alguna medida a la cuantía de recursos que vuelca a fines bélicos, a los que Japón y Alemania Federal destinan cantidades casi despreciables, en cumplimiento de las obligaciones que les fueron impuestas al término de la segunda guerra mundial.

Merece señalarse que la densidad de ingenieros y científicos en el Japón quintuplica el promedio mundial mientras que en los Estados Unidos y Alemania Federal sólo lo triplica, en términos muy aproximados. Si se considerara la densidad de abogados, en cambio, el liderazgo lo ejercería Estados Unidos (279 por cada 100000 habitantes vs. 77 en Alemania Federal y 11 en Japón) ${ }^{2}$. 1987. 
La escasez de recursos naturales constituye un dato estructural en los casos de Japón y Alemania Federal, a diferencia de lo que sucede en los Estados Unidos, generosamente dotado y con un territorio de tamaño continental (cuadro 2). Los dos primeros, en consecuencia, están obligados a lograr una sólida inserción en el comercio internacional de manufacturas, al que los Estados Unidos percibe, en cambio, como un elemento estrictamente complementario y marginal, suscitándole incluso escaso interés la priorización de sectores. Entre los estadounidenses prevalece la percepción, acentuada por la hegemonía economica que ese país ejerció durante cuarenta años, de que el mercado principal es el interno y que si bien la importancia relativa de los distintos sectores puede experimentar cambios en el transcurso del tiempo, el sistema en su conjunto parecía, al menos hasta fines de los años setenta, punto menos que invulnerable. Diversos analistas confirman el predominio en los círculos económicos, políticos y académicos de una visión centrada en la problemática interna (Branson y otros, 1980; Lodge, 1986; Zysman y Tyson, 1983; Oxford Analítica, 1986; Lodge y Vogel, 1987).

La estampida de los precios del petróleo en 1973 representó un peso adicional significativo para los tres países. Sin embargo, en los casos de Japón y Alemania Federal el incremento de la factura energética fue compensado con creces

Cuadro 2

ESTADOS UNIDOS-JAPON-ALEMANIA FEDERAL; SALDOS COMERCIALES POR SECTORES DE ACTIVIDAD ECONOMICA (Millones de dolares)

\begin{tabular}{|c|c|c|c|c|c|c|c|}
\hline & 1970 & 1975 & 1981 & 1982 & 1983 & 1984 & 1985 \\
\hline \multicolumn{8}{|l|}{ Agricultura: } \\
\hline EE.UU. & 631 & 12069 & 25344 & 19728 & 16518 & 13307 & 3659 \\
\hline Japón & -5292 & -13991 & -24929 & -23508 & -23301 & -25776 & $\ldots$ \\
\hline Alemania Federal & -5774 & -10145 & -13441 & -12852 & -12868 & -15568 & $\cdots$ \\
\hline \multicolumn{8}{|c|}{ Indusiria manufacturera": } \\
\hline EE.UU. & 4154 & 21196 & 13369 & -3942 & -28925 & -82377 & -107566 \\
\hline Japón & 13180 & 42393 & 119152 & 107197 & 113403 & 131689 & ... \\
\hline Alemania Federal & 14424 & 39338 & 62317 & 68174 & 59013 & 60235 & $\ldots$ \\
\hline \multicolumn{8}{|l|}{ Energía: } \\
\hline EE.UU. & -1480 & -21922 & -73974 & -54665 & -50349 & -53814 & -45759 \\
\hline Japon & -3858 & -25432 & -72091 & -65306 & -58636 & -59989 & ... \\
\hline Alemania Federal & -1616 & -10286 & -32729 & -29694 & -26694 & -25545 & $\cdots$ \\
\hline \multicolumn{8}{|l|}{ Minerfa: } \\
\hline EE.UU. & -868 & -1295 & -5183 & -3426 & -5298 & -6424 & 1302 \\
\hline Japón & -3698 & -5734 & -11223 & -10388 & -10055 & -10554 & $\cdots$ \\
\hline Alemania Federal & -2343 & -2662 & -3835 & -3651 & -3231 & -571 & ... \\
\hline \multicolumn{8}{|l|}{ Otros sectores: } \\
\hline EE.UU. & 196 & 640 & 758 & -280 & -1268 & 188 & -245 \\
\hline Japón & 105 & 594 & -2168 & -1095 & -877 & -1758 & $\ldots$ \\
\hline AJemania Federal & -318 & -431 & -176 & -712 & 375 & 171 & ... \\
\hline \multicolumn{8}{|l|}{ Total: } \\
\hline EE.UU. & 2638 & 10688 & -39686 & -42585 & -69322 & -129120 & -148609 \\
\hline Japón & 437 & -2110 & 8741 & 6900 & 20534 & 33611 & $\ldots$ \\
\hline Alemania Federal & 4375 & 15814 & [2 142 & 21092 & 16595 & 18722 & $\ldots$ \\
\hline
\end{tabular}

Fuente: División Conjunta craru Jonud, sobre la base de cifras de Naciones Unidas, Intemational Trade Statistics Yearbook, 1970-1971, 1977, 1983, 1984, Commodity Trade Statistics, 1985.

Manufacturas incluye secciones cuci 5 a 8 , menos capítulo 68. 
por el superávit del sector manufacturero. Por el contrario, el déficit energético estadounidense resultó agravado por una erosión nada despreciable - unos 8000 millones de dólares entre 1975 y 1981 - del superávit manufacturero. El desempeño de la industria fue notablemente mejor en Japón y Alemania Federal debido a que estos paises habían generado una base de apoyo que les permitio reaccionar con flexibilidad y prontitud ante las señales que anunciaban el ocaso de la era de la energía barata.

A partir de mediados de los años setenta, las diferencias en cuanto a la evolución del sector manufacturero se acentúan. De esta forma, al promediar la década en curso Japón y Alemania Federal acumulaban en conjunto un superávit comercial cercano ya a los 200000 millones de dólares en el sector manufacturero, en tanto que Estados Unidos registraba un déficit superior a 80000 millones. Los dos primeros países serfan la fuente más importante del superávit manufacturero a nivel mundial; Estados Unidos, por su parte, sería el país cuyo déficit manufacturero habria alcanzado su mayor expresión.

A comienzos de los años setenta, los tres países presentaban superávit relativamente módicos y de órdenes de magnitud comparables, aunque mayores en Japón y Alemania Federal. En apenas 15 años, entonces, su posición relativa ha experimentado un vuelco. Estados Unidos, que a fines de la segunda guerra generaba el $60 \%$ de la producción industrial del mundo, aparece, al promediar los años ochenta, en situación desmedrada precisamente ante los dos países que quedaron en ruinas al término de aquel conflicto.

A comienzos de los años ochenta la inserción internacional de Estados Unidos se torna muy semejante a la de los países latinoamericanos en general. Ella se basa en el sector agrícola, en el cual exhibe un superávit nada despreciable. En todos los demás rubros la economía norteamericana muestra déficit, destacando el del sector manufacturero, de manera que para Estados Unidos, la evolución de los términos de intercambio pasa a convertirse en un tema trascendente.

No hay razones para suponer que desaparecerá la tendencia histórica a la erosión de los términos de intercambio del sector agrícola vis a vis el industrial. En el evento de que se mantuviesen constantes las cantidades exportadas e importa- das de productos agrícolas e industriales, Estados Unidos enfrentaría un deterioro creciente, asociado a la evolución de los términos de intercambio a escala mundial. Así, la preocupación por esta variable - hasta hace algunos años considerada parte del folklore latinoamericano- alcanza ahora al país que lidera la economía mundial.

Hay elevado consenso en cuanto a la existencia de un sólido vínculo entre competitividad, incorporación de progreso técnico, dinamismo industrial y aumento de la productividad. $\mathrm{El}$ incremento de la competitividad constituye un imperativo ineludible en un periodo de transición entre dos patrones tecnológicos y es determinante de los cambios de mediano y largo plazo en la posición relativa de los países dentro de la economía internacional. De allí, entonces, que el esfuerzo que despliegan las naciones desarrolladas por mejorar sus niveles de competitividad en el sector industrial merece para sus respectivos gobiernos una prioridad semejante a la que otorgan a los problemas políticos más cruciales, situación que en el pasado sólo se habia observado en las contingencias bélicas. La importancia asignada en Europa a los distintos programas regionales de cooperación científica o técnica así lo testimonia.

Menos consenso se advierte en cuanto a la forma de medir la competitividad y, mucho menos aún, respecto a cómo lograr que ésta se incremente. Hay acuerdo en el sentido de que la erosión de la productividad, iniciada hace un par de décadas y que se ha acentuado, sobre todo en los Estados Unidos, desde la segunda mitad de los años setenta, encierra graves consecuencias potenciales. Sin embargo, hondas son las discrepancias respecto del porqué de este deterioro $y$, por consiguiente, de los mecanismos más eficientes para revertirlo.

En los siete indicadores alternativos de competitividad internacional considerados en este trabajo, la posición relativa de los tres paises es la misma: primero Japón; después Alemania Federal y, último, los Estados Unidos (cuadro 3).

El esfuerzo de investigación y desarrollo para fines civiles es significativamente mayor en Japon y Alemania Federal, y en diversos estudios se menciona este hecho como una posible causa del distinto ritmo de evolución de la competitividad en los tres países. De otro lado, el dinamismo de 
Cuadro 8

COMPETITIVIDAD INTERNACIONAL: DIFERENTES INDICADORES

\begin{tabular}{|c|c|c|c|}
\hline & $\begin{array}{l}\text { Estados } \\
\text { Unidos }\end{array}$ & Japón & $\begin{array}{c}\text { Alemania } \\
\text { Federal }\end{array}$ \\
\hline Gastos en l y D/PIB (1983-84) & $\begin{array}{l}1.8 \\
(9)\end{array}$ & $\begin{array}{l}2.5 \\
(1)\end{array}$ & $\begin{array}{l}2.4 \\
(2)\end{array}$ \\
\hline \% crecimiento exportación & & & \\
\hline manufacturas 1983-1969 & $\begin{array}{l}7.9 \\
(3)\end{array}$ & $\begin{array}{l}18.4 \\
(I)\end{array}$ & $\begin{array}{l}9.3 \\
(2)\end{array}$ \\
\hline $\begin{array}{l}\text { Exportación bienes capital/exportación total } \\
\text { manufacturas, } 1983(\%)\end{array}$ & $\begin{array}{l}44 \\
(3)\end{array}$ & $\begin{array}{l}58 \\
(1)\end{array}$ & $\begin{array}{l}46 \\
(2)\end{array}$ \\
\hline $\begin{array}{l}\text { Exportación bienes capital/exportación } \\
\text { mundial bienes capital (\%) 1983-1963 }\end{array}$ & $\begin{array}{l}74 \\
(3)\end{array}$ & $\begin{array}{r}475 \\
(1)\end{array}$ & $\begin{array}{r}100 \\
(2)\end{array}$ \\
\hline \multicolumn{4}{|l|}{$\begin{array}{l}\text { Exportación bienes capital/importación bie- } \\
\text { nes capital }(\%)\end{array}$} \\
\hline 1983 & 100 & 950 & 267 \\
\hline 1963 & $\begin{array}{r}383 \\
(3)\end{array}$ & $\begin{array}{r}200 \\
\text { (1) }\end{array}$ & $\begin{array}{r}380 \\
(2)\end{array}$ \\
\hline \multicolumn{4}{|l|}{$\begin{array}{l}\text { Crecimiento (\%) productividad manufactu- } \\
\text { rera }\end{array}$} \\
\hline $\begin{array}{l}1975-1981 \\
1965-1973\end{array}$ & $\begin{array}{l}1.7 \\
2.8 \\
(3)\end{array}$ & $\begin{array}{r}8.7 \\
11.0 \\
\text { (I) }\end{array}$ & $\begin{array}{c}3.2 \\
4.2 \\
\text { (2) }\end{array}$ \\
\hline $\begin{array}{l}\text { Exportaciones manufactureras/importacio- } \\
\text { nes manufactureras }\end{array}$ & & (1) & \\
\hline $1979-1981$ & 1.0 & $\begin{array}{l}5.0 \\
(1)\end{array}$ & $\begin{array}{l}1.8 \\
(2)\end{array}$ \\
\hline
\end{tabular}

Fuente: División Conjunta CEPALONNUDh, Global compatition, p. 100; Naciones Unidas, Bulletin of statistics on world trade in engineering products, 1983; World Bank Report, Productivity in industry, (KDE, 1986.

las exportaciones industriales japonesas es avasallador en las últimas dos décadas; su ritmo de crecimiento duplica el de las ventas al exterior de los otros dos países. La primacía nipona se hace sentir, también, en la significación que alcanzan dentro de las exportaciones manufactureras los productos de mayor contenido tecnológico. No es extraño, entonces, que en 1983 la importancia relativa de Alemania Federal dentro de las ventas mundiales de esos productos sea la misma que veinte años antes, la de Estados Unidos equivalga a sólo el 74\% y la del Japón casi se quintuplique.

El siguiente indicador utilizado en este ejercicio se refiere más específicamente a la competitividad de los bienes denominados engineering products que son los que tienen un alto contenido de tecnología moderna, como se ha mencionado. La relación exportaciones/importaciones de este tipo de bienes, que en 1963 era de casi 4:1 en las economias estadounidense y oestealemana, cae sustancialmente en ambas en 1983, aunque con mayor fuerza en la primera. En el caso japonés, en cambio, la relación casi se quintuplica en ese lapso.

El ritmo de incremento de la productividad, factor determinante en la evolución de la competitividad a largo plazo, se debilita a partir de mediados de la década de 1970. El fenómeno alcanza mayor intensidad en los Estados Unidos, país donde el mejoramiento había sido más lento en el periodo anterior. El ritmo de crecimiento de la productividad en los dos subperíodos considerados aquí es más alto en Japón, siguiendo después Alemania Federal, con lo que se mantiene la constante observada en todos los indicadores (cuadro 3).

Este ordenamiento se da no sólo en cuanto al nivel, sino también en lo que respecta a la trayectoria de la competitividad, según se desprende del cotejo de la relación exportaciones/importaciones de manufacturas en el trienio 1979-81. Para Japón ella es 5, no llega a 2 para Alemania Federal y alcanza apenas a 1 para los Estados Unidos. 
III

\section{Factores condicionantes de la competitividad internacional}

Desde una perspectiva de mediano y largo plazo, la competitividad consiste en la capacidad de un país para sostener y expandir su participación en los mercados internacionales, y elevar simultáneamente el nivel de vida de su poblacion. Esto exige el incremento de la productividad $y$, por ende, la incorporación de progreso técnico.

La experiencia internacional enseña que no existe "otro sendero" para conseguir un mejoramiento sólido de la competitividad de un país. Es efectivo que en el corto plazo la devaluación de la moneda nacional mejora la posición relativa de sus empresas. Sin embargo, este recurso es de limitada eficacia, puesto que por sí solo no incrementa la productividad ni estimula la incorporación de progreso técnico. Por el contrario, tiende a erosionar la cohesión social, lo que a la postre atenta contra la viabilidad de una inserción internacional más eficiente. Es natural que las empresas procuren incrementar su competitividad internacional sobre la base de aprovechar la disponibilidad de mano de obra de bajo costo y de líneas subsidiadas de crédito, y de compensar los márgenes reducidos e incluso negativos en el mercado externo con utilidades elevadas obtenidas en el mercado interno protegido, o de aprovechar franquicias tributarias especiales, etc. De esta forma talvez logren utilidades satisfactorias, las que, sin embargo, tendrán poco que ver con un aumento de la competitividad del pais, entendida ésta en un sentido amplio, por más que se observen también mejoras en el balance comercial y en el coeficiente de exportaciones.

Desde una perspectiva estrecha, puede aducirse que América Latina ha logrado avances importantes en materia de competitividad internacional durante los años ochenta. Pero ellos resultan espúreos cuando se adopta un enfoque más integral, toda vez que se dan en presencia de una caída del ingreso por habitante, una merma de los coeficientes de inversión, rebaja del gasto en investigación y desarrollo tecnológico y en el sistema educativo, y erosión de los salarios reales.

Lo anterior no significa desconocer que en los últimos años algunos paises o sectores han logra do incrementos "auténticos" de la competitividad - en contraste con lo que sería una elevación "espúrea"-, basados en mejoras de la productividad con incorporación de progreso técnico. Este fenómeno constituye un valioso precedente para el logro de una efectiva modernización del aparato productivo.

El apreciable incremento del excedente comercial de muchos paises de la region ha apuntado únicamente a sustentar la cuantiosa transferencia de recursos financieros exigida por el servicio de la deuda externa, por lo que no ha logrado satisfacer algunos requisitos indispensables de una auténtica modernización. No cabe confundirlo, entonces, con el promisorio comienzo de un proceso de sostenida y sólida elevación de la competitividad del aparato productivo latinoamericano.

Ya se señaló que en el corto plazo el único instrumento de política que puede afectar rápida y significativamente la competitividad de un país es el tipo de cambio. Sin embargo, un análisis que considere un horizonte de mediano plazo detectará tendencias divergentes en la posición relativa de las naciones industrializadas en el comercio internacional de productos manufacturados.

Lo sucedido en los anios ochenta, con las erráticas fluctuaciones del dólar -brusco ascenso hasta 1985, caida posterior-, comprueba que, no obstante las acentuadas variaciones de los flujos comerciales, persisten las tendencias de largo plazo, esto es la erosión de la competitividad industrial de los Estados Unidos, el ascenso sistemático de Japón, y el leve aumento de Alemania Federal. Debe concluirse, por tanto, que las diferencias en la inserción internacional obedecen en medida importante a factores de carácter estructural que afectan, incluso, las modalidades y los resultados de las estrategias nacionales, $y$ al uso que cada país hace de los instrumentos específicos de política económica e industrial.

A continuación se intenta identificar algunos de los factores que explican la diferente competitividad de los paises en el sector industrial. 
Cuadro 4

COEFIGIENTE DEUDA/VALOR COMERCIAL DE LOS ACTIVOS DEL SECTOR EMPRESARIAL NO FINANCIERO

(Porcentajes)

\begin{tabular}{|c|c|c|c|c|c|c|c|c|}
\hline Países & $1966-1973$ & $1974-1979$ & 1980 & 1981 & 1982 & 1983 & 1984 & 1985 \\
\hline Estados Unidos & 0.54 & 0.96 & 0.77 & 0.92 & 0.87 & 0.78 & 0.90 & 0.83 \\
\hline Japán & 3.08 & 3.31 & 3.14 & 2.91 & 2.92 & 2.68 & 2.11 & $1.82^{\circ}$ \\
\hline Alemania Federal ${ }^{b}$ & 2.38 & 3.36 & 3.85 & 4.13 & 4.11 & 3.48 & 3.42 & 2.39 \\
\hline Francia & $1.17^{x}$ & 1.33 & 1.29 & 1.40 & 1.55 & 1.56 & $\ldots$ & $\ldots$ \\
\hline Reino Unido & 0.67 & 1.38 & 1.19 & 1.23 & 1.09 & 0.87 & 0.74 & $0,70^{\mathrm{a}}$ \\
\hline
\end{tabular}

Fuente: División Conjunta CEPAL/ONud, sobre la base de Banco de Pagos Internacionales, Fif fy-Six Annual Report, abril 1985-maszo 1986.

a Estimados.

b Todas las empresas, excluido sector habitacional.

c 1970-1973.

\section{El ritmo de inversion}

El coeficiente de inversión reviste gran importancia en la explicación del incremento de productividad (Denison, 1980). Los países cuya tasa de inversión se queda a la zaga, ven debilitarse el ritmo de crecimiento de su productividad $y$, por consiguiente, de su competitividad, según lo muestra la experiencia de Estados Unidos y el Reino Unido en las últimas tres décadas. Japón y los nuevos países industrializados del Asia comprueban que el incremento de la tasa de inversión se traduce en mejoras apreciables de la competitividad.

\section{La asignación de recursos para inversión}

La reestructuración productiva supone el desplazamiento intersectorial de considerables volúmenes de recursos de inversión, proceso en el que intervienen las empresas, el sistema financiero y el sector público, y que en cada país toma perfiles propios, según la importancia relativa de los diferentes actores y sus vinculaciones recíprocas (Zysman, 1984).

En los Estados Unidos y el Reino Unido, el nivel de endeudamiento de las empresas con el sistema financiero es sustancialmente inferior al que prevalece en Japón, Alemania Federal y Francia (cuadro 4). En los primeros, el mercado de capitales determina el destino de los ahorros, incluidos los generados en las empresas, los que pueden ir a la inversión, la especulación financiera o el crédito para el consumo de las familias. En los otros países, en cambio, un determinado porcentaje de los recursos de inversión (grandes proyectos) se canaliza en función de prioridades sectoriales definidas institucionalmente por la banca o el sector público, es decir en forma autónoma de quienes han generado la contrapartida de ahorros (cuadro 5).

\section{El Mercado de trabajo y el "Estado de bienestar"}

La reestructuración productiva exige desplazamientos que entrañan inexorablemente elevados costos, humanos y económicos. Esto confiere gran trascendencia al tema de las "rigideces del mercado de trabajo", lo que ha conducido al cuestionamiento del "Estado de bienestar" (Pfaller, 1987; Dande Stadt, 1987).

Cuadro 5

TIPOLOGIA DE SISTEMAS

FINANCIERO-INDUSTRIALES

\begin{tabular}{ll}
\hline Pafses & $\begin{array}{l}\text { Sistema financiamiento industrial } \\
\text { Regulación de crédito con precios } \\
\text { Jadministrados }\end{array}$ \\
República Federal & $\begin{array}{l}\text { Regulación de crédito por parte } \\
\text { de la banca }\end{array}$ \\
Alemana & Mercado de capitales. \\
Entados Unidos-Reino & \\
\hline
\end{tabular}

Fuente: J. Zysman, Governments, markets and grototh: financial systems and the politics of indtastrial change, Cornell University Press, 1983. 
En las décadas de crecimiento y prosperidad se fue generando una institucionalidad que complementaba y atenuaba los resultados del libre juego de las fuerzas del mercado. Mediante la fijacion de salarios mínimos, la vigencia de mecanismos de indización de las remuneraciones, el otorgamiento de subsidios de desempleo y otros beneficios de seguridad social, la materialización de programas de capacitación, la concesión de subsidios regionales, esta institucionalidad brindaba protección y apoyo a los estratos sociales, sectores productivos y zonas geograficas en situación relativamente desmedrada. La incesante expansión de este esquema, cuyo costo era financiado en parte por las empresas, habría deteriorado seriamente la eficiencia microeconómica-social, tornándose incompatible con los imperativos de la competitividad internacional.

El problema estriba en que ese "Estado de bienestar" generaba también beneficios desde el punto de vista macroeconómico-social, los cuales cobran especial trascendencia en coyunturas de cambio estructural. Entre ellos destacan la legitimidad social de las instituciones, que favorece la cohesión social, y la existencia de un avanzado sistema educativo y, por consiguiente, de una mano de obra altamente calificada. Puede agregarse que es dificil aprehender mediante indicadores agregados el verdadero carácter y magnitud del "Estado de bienestar". Más importante que el peso cuantitativo de las instituciones públicas es el tipo de relación que se entabla entre ellas y los sectores empresarial y laboral. Por lo demás, no obstante el auge de la retórica "prekeyseniana", el peso cuantitativo del sector público y de la seguridad social en los países industrializados no ha disminuido (cuadros 6,7 y 8 ).

Las diferencias más de fondo se dan entre Japón y los Estados Unidos, pese a que en ambos la gravitación económica del sector público es relativamente baja, a diferencia de lo que sucede en los países europeos. Ellas obedecen a las contrapuestas modalidades de articulación entre los sectores público y privado, las cuales se manifiestan en marcadas diferencias en el uso de los instrumentos de politica por parte de las autoridades estadounidenses y niponas.

El mercado laboral europeo se ha flexibilizado significativamente durante la década en curso. La indización salarial se ha corregido (Italia, Bélgica y Francia) o abolido (Dinamarca); se ha estimulado el trabajo temporal (Francia y Alemania Federal); han disminuido los subsidios de cesantía respecto a los salarios medios (Dinamarca y Reino Unido), así como las contribuciones a la seguridad social (Francia, Dinamarca, Bélgica y Reino Unido). De otro lado, se favorece el esquema de jornada parcial y jubilación adelantada (Paises Bajos, Bélgica y Alemania Federal) y se procura erosionar administrativamente (Reino Unido) el papel de los sindicatos en la negociación salarial (BIs, 1986). Sin embargo, el ef́ecto agregado de este proceso de "flexibilización" paulatina no ha sido, como hubiera podido imaginarse, una merma en la significación del sector público en la economía.

\section{Las relaciones industriales}

Existe creciente consenso en cuanto a la influencia que las relaciones industriates gerencialeslaborales a nivel de planta, sector industrial y nivel nacional ejercen en la productividad. No obstante las diferencias en las modalidades institucionales, se verifica sistemáticamente que la menor conflictibilidad en las relaciones favorece los aumentos de productividad. Así lo muestra la experiencia de Japón y, en general, de los países de Europa occidental, que contrasta con la de Estados Unidos y el Reino Unido, donde el carácter de las relaciones industriales sería más conflictivo.

El tema cobra mayor relevancia en un periodo de reestructuración productiva, en que se está gestando un nuevo patrón tecnológico que requiere la cooperación constructiva de los distintos actores económicos, sociales y políticos con el objeto de "absorber" y distribuir el costo del ajuste estructural en curso (Piore, 1986; Brown y Bennett, 1986).

\section{La organizacion empresarial}

La intensificación de la competencia internacional, el surgimiento de un nuevo patrón tecnológico y los rápidos cambios en las preferencias del mercado explican la marcada tendencia a innovar que se observa, tanto en el interior de la estructura organizativa empresarial, como en la naturaleza de las relaciones interempresariales. En ambos casos las relaciones jerárquicas verticales van siendo sustituidas por las de cooperación de carácter horizontal. El criterio básico es el 
logro de flexibilidad, que haga posible la oportuna incorporación de innovaciones tecnológicas y la adaptación a las cambiantes condiciones de la demanda, en un contexto de creciente competencia internacional.

Se parte de la premisa de que la cooperación y el compromiso de quienes laboran en los distintos niveles de la empresa constituyen un factor determinante de los incrementos de productividad. Ello abarca desde la fase de diseño hasta la de control de calidad (Arnold y Ken, 1987; Drucker, 1987a y 1987b); desde el punto de vista del

Cuadro 6

EMPLEO EN LA ADMINISTRACIÓN PÚBLICA COMO PORCENTAJE DEL EMPLEO TOTAL

\begin{tabular}{|c|c|c|c|c|c|c|c|c|c|}
\hline \multirow[b]{2}{*}{ Paises } & \multirow[b]{2}{*}{1960} & \multirow[b]{2}{*}{1975} & \multirow[b]{2}{*}{1980} & \multirow[b]{2}{*}{1985} & \multicolumn{5}{|c|}{ Promedio } \\
\hline & & & & & $1960-1967$ & $1968 \cdot 1973$ & $1974-1979$ & $1980-1985$ & $1960-1985$ \\
\hline Estados Unidos & 15.7 & 17.8 & 16.5 & 15.8 & 16.7 & 17.8 & 17.0 & 16.2 & 19.9 \\
\hline Japon & $\ldots$ & 6.5 & 6.7 & 6.4 & $\ldots$ & $\ldots$ & 6.5 & 6.6 & $\ldots$ \\
\hline Alemania Federal & 8.0 & 13.9 & 14,9 & 16.0 & 9.4 & 11.6 & 14.2 & I5.6 & 12.4 \\
\hline Francia & 13.1 & 14.3 & 15.6 & 17.8 & 12.9 & 13.4 & 14.7 & 16.7 & 14,3 \\
\hline Reino Unido & 14.8 & 20.8 & 21.1 & 21.8 & 15.5 & 18.5 & 20.9 & 21.9 & 18.9 \\
\hline Italia & 8.7 & 14.0 & $\mathbf{I 5 . 0}$ & 15.8 & 9.9 & 12.3 & 14.5 & 15.5 & 12.8 \\
\hline
\end{tabular}

Fuente: División Conjunta CEPAL/ONUDI, sobre la base de OCDE, Economic outlook, Historical statistics 1960-1985.

Cuadro 7

GASTO PUBLICO TOTAL COMO PORCENTAJE DEL PIB

\begin{tabular}{|c|c|c|c|c|c|c|c|c|c|}
\hline \multirow[b]{2}{*}{ Paises } & \multirow[b]{2}{*}{1960} & \multirow[b]{2}{*}{1975} & \multirow[b]{2}{*}{1980} & \multirow[b]{2}{*}{1985} & \multicolumn{5}{|c|}{ Promedio } \\
\hline & & & & & $1960-1967$ & $1968-1973$ & $1974-1979$ & $1980 \cdot 1985$ & 1960-1985 \\
\hline Estados Unidos & 27.0 & 34.6 & 33.7 & 96.7 & 28.3 & 31.0 & 32.6 & 35.6 & 31.6 \\
\hline Jарón & $\ldots$ & 27.3 & 32.6 & 32.7 & $\ldots$ & 20.2 & 28.4 & 33.3 & 26.1 \\
\hline Alemania Federal & 32.4 & 48.9 & 48.3 & 47.2 & 35.7 & 89.8 & 47.5 & 48.4 & 42.3 \\
\hline Francia & $\$ 4.6$ & 49.5 & 46.4 & 52.4 & 87.4 & 39.0 & 43.7 & 50.6 & 42.3 \\
\hline Reino Unido & $\$ 2.3$ & 46.3 & 45.1 & $47.8^{2}$ & 34.7 & 39.9 & 44.4 & $47.0^{\mathrm{\alpha}}$ & 40.8 \\
\hline Italia & 30.1 & 43.2 & 46.1 & 58.4 & 31.9 & 36.0 & 42.9 & 54.2 & 40.5 \\
\hline
\end{tabular}

Fuente: División Conjunta CEPAd ONudr sobre la base de OCDE, Economic outlook, Historical statistics 1960-1985.

d 1984.

Cuadro 8

GASTO EN SEGURIDAD SOCIAL COMO PORCENTAJE DEL PIB

\begin{tabular}{lrrrrrrrrrr}
\hline & & & & & \multicolumn{5}{c}{ Promedio } \\
\cline { 8 - 10 } Paises & 1960 & 1975 & 1980 & 1985 & $1960-1967$ & $1968-1973$ & $1974-1979$ & $1980-1985$ & $1960-1985$ \\
\hline Estados Unidos & 5.0 & 11.1 & 10.9 & 11.0 & 5.4 & 7.7 & 10.3 & 11.3 & 8.4 \\
Japón. & 3.8 & 7.7 & 10.1 & 11.0 & 4.1 & 4.8 & 8.4 & 10.8 & 6.8 \\
Alemania Federal & 12.0 & 17.6 & 16.5 & 16.1 & 12.4 & 13.2 & 26.7 & 16.8 & 14.6 \\
Francia & 13.5 & 20.4 & 23.2 & 26.4 & 15.5 & 17.2 & 21.0 & 25.4 & 19.4 \\
Reino Unido & 6.8 & 9.9 & 11.5 & $14.0^{2}$ & 7.3 & 8.8 & 10.5 & $13.2^{\text {a }}$ & 9.6 \\
ltalia & 9.8 & 15.6 & 15.8 & 19.5 & 11.1 & 19.0 & 15.4 & 18.5 & 14.2 \\
\hline
\end{tabular}

Fuente: División Conjunta CEPAL/ONUDI, sobre la base de OCDE, Economic outlook, Historical statistics, 1960-1985.

a 1984. 
esquema organizativo, exige reducir el número de escalones jerárquicos y reforzar la vinculación horizontal en cada uno de los niveles.

La cooperación entre empresas, que adquiere modalidades muy diversas, ha tendido a intensificarse, en un contexto de creciente unificación del mercado internacional, en sus componentes de oferta, demanda y acervo tecnológico. A continuación, algunas de las expresiones más interesantes de este fenómeno.

i) Empresas-redes. En virtud de acuerdos flexibles, ensambladores, proveedores, comercializadores y centros de investigación tecnologica, se vinculan con instancias centrales de coordinación para los aspectos financieros, publicitarios y de estrategia corporativa.

ii) Esquemas de cooperación en el plano de la investigación y el desarrollo entre empresas y gobiernos europeos (programa EUREKA).

iii) Esfuerzos cooperativos en investigación y desarroilo y coproducción entre las empresas del sector automotor de los Estados Unidos, Japón y Europa.

iv) Esquemas cooperativos de investigación y desarrollo en las empresas productoras de semiconductores en los Estados Unidos, con énfasis en el área de ingeniería de producción. Acuerdos de esta naturaleza son habituales y de antigua data en Japón, bajo el liderazgo del MITI; tal vez la experiencia más significativa haya sido el programa de veinte años de duración con el que se enfrentó el "desafío ивм".

v) En los sectores más expuestos a la "moda", se observan esquemas en los que coexisten la competencia —en la fase de la exhibición de los modelos-y la cooperación en la fase de producción, una vez que el mercado ha definido los "ganadores".

A su turno, la proliferación de los esquemas de cooperación entre las firmas multinacionales obedece, en parte, al creciente costo de desarrollo de productos y procesos, así como al imperativo de adaptarse a las súbitas modificaciones en las paridades cambiarias. Las multinacionales parecen haberse percatado de que en la actualidad el conocimiento tecnológico y las calificaciones profesionales están distribuidas en forma bastante homogénea entre los países industrializados; en consecuencia, cualquier fase del proceso productivo puede materializarse en cualquier lugar. Les resulta conveniente, entonces, instalar plan- tas o suscribir acuerdos con empresas de otros paises en las distintas regiones, lo cual ayuda asimismo a sortear las eventuales barreras proteccionistas.

\section{La infraestructura educacional $y$ de investigación y desarrollo}

Existe unanimidad en cuanto a que este aspecto constituye un requisito e ingrediente primordial de toda reestructuración productiva que lleve aparejado progreso técnico. Esto explica el incremento, desde fines de la década de 1970, de los recursos asignados a investigación y desarrollo en las naciones industrializadas, así como la toma de conciencia de que es imprescindible readecuar el sistema educativo, a fin de adaptarlo a las nuevas necesidades.

Un interesante elemento de diferenciación entre los patses tiene que ver con la magnitud de los recursos para investigación y desarrollo asignados a la industria bélica y la evaluación de su impacto en el ámbito de la competítividad. La polémica acerca del efecto colateral de estas inversiones en el conjunto del sector industrial no está agotada. Parecería que, al menos en determinados plazos, pueden coexistir niveles bajos de competitividad internacional en los productos transables, con niveles de excelencia en la esfera militar, acaso porque se trata de actividades cuyos desafíos, procedimientos, plazos y formas organizativas difieren radicalmente. En la esfera militar se privilegia la definición de objetivos, metas, antes que de plazos, y la restricción económica desempeña un papel notoriamente inferior. La posibilidad de programar a largo plazo está muy lejos de reproducirse en el ámbito industrial, comercial, donde lo esencial es la flexibilidad y la capacidad de rápida adaptación a las cambiantes tendencias del comercio internacional. Más aún, en el ámbito militar la competencia es menos ardua y los plazos son más amplios. El reemplazo de generaciones sucesivas de "productos" y la "diferenciación" dentro de cada generación no están determinados, afortunadamente, por la prueba de su desempeño en el uso de las mismas. La esfera militar posee la capacidad de atraer los talentos más notables de la ciencia y la tecnologia, ya que puede otorgarles un ambiente seguro y tranquilo, donde, aparte de remuneraciones elevadas, no se les presiona 
para que su trabajo genere resultados en el corto plazo.

Se explica, entonces, que el grupo de países desarrollados que ha canalizado recursos significativos a la esfera militar acuse precarios niveles de competitividad industrial en los productos convencionales; y que el grupo de países con elevado grado de industrialización, pero que prácticamente no destina recursos a fines de defensa, tenga el liderazgo en la competitividad industrial internacional de aquellos productos.

A mediados de la década en curso, el superávit manufacturero de Japón, Alemania Federal e Italia, los tres derrotados en la última conflagración mundial, bordeaba los 220.000 millones de dólares. Por el contrario, las tres potencias vencedoras acumulaban un déficit cercano a los 120.000 millones de dolares, el $75 \%$ de los cuales correspondía a los Estados Unidos. En términos generales, los países militarmente derrotados en la segunda guerra mundial abastecen el déficit tanto de quienes fueron vencedores como del resto del mundo y, fundamentalmente, de las naciones en desarrollo.

Se detecta una inequívoca relación inversa entre importancia de los gastos en defensa dentro del PIB y el grado de competitividad internacional, medido éste en términos de la significación del superávit o déficit manufacturero respecto del producto manufacturero (gráfico 1 ).

\section{Gráfico 1}

\section{COMPETIVIDAD INTERNACIONAL Y GASTOS EN DEFENSA}

(Promedio 1981-1983)

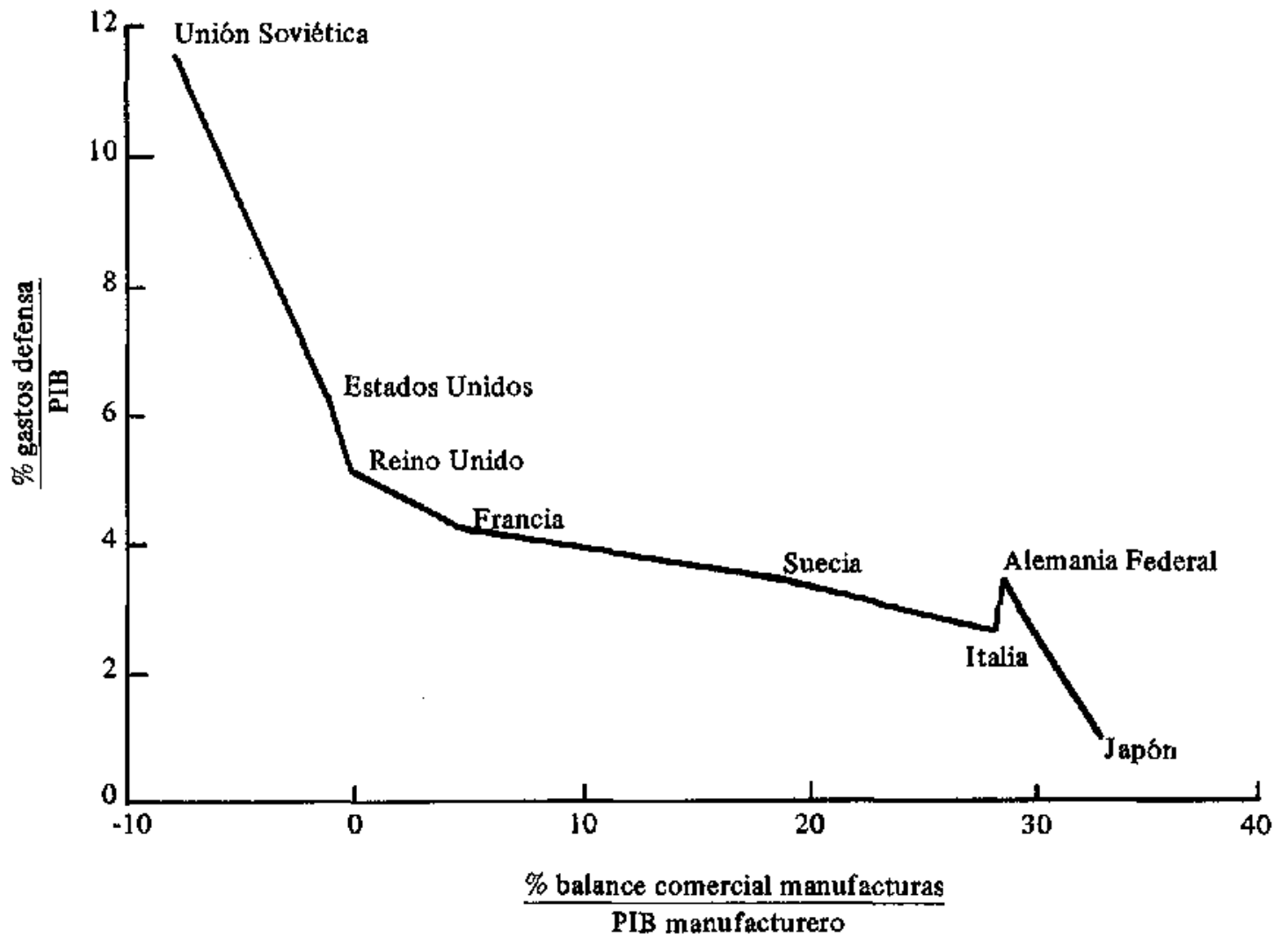

Fuente: División Conjunta CEPAL/ONUDI de Industria y Tecnología, a base de información de SIPRI, World Armaments and Disarmament, Anuario 1985. 
La Unión Soviética y los Estados Unidos se sitúan en un extremo y Alemania Federal y Japón en el otro, quedando el Reino Unido, Francia, Suecia e Italia en las posiciones intermedias. El efecto multiplicador del gasto de defensa en la competitividad industrial internacional sería negativo, a despecho de lo que postula la sabiduría convencional.

Un acuerdo entre los Estados Unidos y la Unión Soviética en favor del desarme permitiría liberar una fracción considerable de los recursos que destinan a fines bélicos. Y en el caso de que este gráfico describiese adecuadamente la evolución del desempeño para cada país, ellos dos "recorrerian la curva" en la dirección de la elevación de la competitividad industrial, factor clave para enjugar sus respectivos déficit externos. Tal acuerdo, en consecuencia, suscitarfa no sólo un benéfico impacto sobre la ominosa percepción colectiva acerca del futuro de la humanidad, sino que favorecería la recuperación de los equilibrios en los flujos comerciales y financieros.

\section{La estructura sectorial y la absorcion de progreso técnico}

Es sorprendente la relación positiva que se observa entre la ausencia de recursos naturales y el nivel de competitividad en el sector industrial. Aquellos países que carecen de la fuente "fácil" de generación de divisas, que constituyen los recursos naturales, no tienen otra alternativa que optar por la "construcción" de ventajas comparativas en el sector manufacturero. Aleccionadoras son en tal sentido la experiencia de Japón, Alemania Federal e Italia. Ellas contrastan con las de Estados Unidos, la Unión Soviética y el Reino Unido, países bien dotados de recursos naturales o que tenían asegurado su abatecimiento a bajos precios descle las colonias.

Los países nórdicos protagonizan un caso especial, en el que coexisten una generosa disponibilidad de recursos naturales y una alta competitividad derivada de elevados niveles de especializacion en equipos y procesos vinculados a la explotación, procesamiento y elaboración de tales riquezas (agricultura, silvicultura, pesca y energía, en el caso de Noruega). Se trata de paises pequeños, para los cuales la especialización es un imperativo y en los que el "Estado de bienestar" coexiste con una gran apertura al comercio exte- rior. Además, persiste en ellos el criterio de que la búsqueda del pleno empleo no compromete necesariamente la competitividad, cuya salvaguardia es a su turno requisito para mantener los niveles de prosperidad. En Suecia, Finlandia y Noruega la tasa de desempleo se mantiene en el rango del $3 \%$, en tanto que el promedio para Europa occidental en su conjunto bordea el $10 \%$.

Italia ofrece una experiencia peculiar de reestructuración productiva, ya que se aparta del patrón predominante -que se sustenta en los ejes electrónico y químico- impuesto por los Estados Unidos, Japón y Alemania Federal. Aparte de registrar avances significativos en estas áreas intensivas en contenido tecnológico, Italia logra mantener su inserción internacional, mediante la elevación de los niveles de excelencia en sectores que, desde el punto de vista tanto del "sentido común" como del académico —coincidencia poco frecuente-aparecían condenados a la condición de "perdedores", a causa de la creciente competitividad de los NIc's asiáticos (Piore y Sabel, 1983 y 1984). De esta forma, las ramas textil, vestuario y calzado, que en los demás paises industrializados fueron virtualmente desmanteladas, mantienen en Italia un vigoroso dinamismo y logran una elevada productividad, que les permite consolidar posiciones no sólo en el Mercado Común Europeo, sino también en el estadounidense (Piore y Sabel, 1983 y 1984; Ribeiro y otros, 1987).

La pequeña y mediana industria desempenan en Italia un papel más importante, cuantitativamente, que en el resto de los países europeos considerados. En particular, que en Alemania Federal, Suecia, Reino Unido y Francia, Mayor significación reviste el incremento medio de la productividad que exhibe este sector a partir de la década de 1970 , variable que en una gama relativamente amplia de agrupaciones industriales, lega de esta forma a asemejarse a la imperante en la gran empresa. Ello refuta la imagen convencional de que entre ambos sectores hay diferencias estructurales, vale decir insalvables, de productividad, asociadas a economias de escala y rigideces tecnologicas. El mencionado fenómeno permitio que Italia alcanzara una posición nada despreciable en rubros tales como textiles, vestuario, calzado, muebles de madera y ciertos tipos de maquinaria -en especial para usos específicos, particularmente para el rubro alimenta- 
rio-, en los cuales las economías de escala no son significativas.

La modernización de sectores tradicionales, supuestamente destinados a desaparecer en forma inexorable, y la elevación significativa de la productividad en la pequeña y mediana empresa en una amplia gama de sectores son peculiaridades que confieren particular trascendencia al caso italiano para la reflexión sobre las opciones que se abren a las naciones latinoamericanas, lo cual no implica, por cierto, otorgarle un carácter paradigmático.

La espectacular reestructuración italiana de la última década se explica sólo parcialmente por la necesidad de compensar la presión emanada de las organizaciones laborales que adoptan como referente los niveles salariales imperantes en los sectores de mayor densidad de capital (automotor, química y siderurgia). En consecuencia, el aumento de la productividad no sólo sería compatible con la presión salarial, sino que, hasta cierto punto, habría sido inducida por ésta. Se trata de un ejemplo concreto del enfoque que asocia el incremento de la competitividad a la elevación de la productividad y al progreso técnico (Antonelli, 1987).

En Francia, cuyo crecimiento industrial en el período es sólo levemente inferior al de Alemania Federal e Italia, se observan cambios en el perfil productivo industrial que también se apartan de los casos clásicos de los tres países mayores. El liderazgo lo ejerce el sector de maquinaria eléctrica y electrónica. En forma simultánea, otras agrupaciones, algunas de ellas de uso intensivo de mano de obra, otras de recursos naturales o bien de capital físico - minerales no metálicos, siderurgia, minerales no ferrosos, productos metálicos, textiles, cuero y madera- experimentan lo que se ha dado en denominar un proceso de desindustrialización. El resultado global es un acelerado crecimiento de las actividades manufactureras, un acentuado cambio estructural, una especialización en torno al eje de equipo eléctrico y electrónico -energía nuclear, aeronáutica, equipo ferroviario, telecomunicaciones y armamento-, proceso que es vigorosamente apoyado merced al empleo del poder de compra del sector público (Messine, 1984 y 1985; McCormick, 1987; Boyer, 1983a, 1983b; Boyer y Mistral, 1983; Lodge y Vogel, 1987).

\section{El uso de instmumentos de politica y la dimensión institucional}

Para ilustrar la importancia de las especificidades nacionales del diseño y uso de instrumentos de política que inciden en el sector industrial, se aborda enseguida, esquemáticamente, un análisis comparativo entre Japón y los Estados Unidos.

Igual que el resto del mundo, Japón adopta como referente el patrón de consumo prevaleciente en los Estados Unidos. Lo hace con el propósito fundamental de generar los mismos bienes que ese país demanda, pero en condiciones más favorables de costo y calidad. Estados Unidos se transforma así en el objetivo principal al cual se dirige la estrategia nipona de producción y exportación. Sin embargo, en el ámbito interno, las autoridades japonesas adoptan una serie de resguardos para impedir que la difusión, en todo caso gradual, de ese patrón dificulte el logro de las metas fundamentales de crecimiento que el país se ha planteado. Se reproduce la "modernidad", pero se cautela su tasa de absorcion, de modo de compatibilizarla con el objetivo estratégico del crecimiento interno $y$, por tanto, del avance en materia de competitividad.

El automóvil y la vivienda residencial cumplen, en términos tanto cuantitativos como cualitativos, un papel crucial en el patrón de consumo de los estadounidenses. No es extraño que la significación de estos elementos en el consuma familiar haya venido aumentando sistemáticamente en el transcurso del tiempo, de la mano con el crecimiento del ingreso. Pues bien, las autoridades niponas adoptan medidas para dificultar o retardar la reproducción de ese esquema de consumo en su mercado interno. Para tal efecto, aplican una política destinada de manera sistemática a comprimir el consumo y estimular el ahorro, en lo concerniente tanto a la vivienda como a la adquisición de bienes de consumo, principalmente los duraderos. Mientras en los Estados Unidos los intereses generados por las cuentas de ahorros son sujetos de impuesto y los intereses que se pagan por concepto de crédito al consumo están exentos de gravámenes tributarios, en Japón sucede exactamente lo contrario. En el caso específico de la vivienda, las instituciones que proveen financiamiento en los Estados Unidos se hallan en condiciones de of recer una 
tasa de interés inferior a la del sistema bancario, aparte de que los intereses que se pagan por concepto de la deuda habitacional están exentos de tributos, aun cuando se trate de la segunda o incluso la tercera casa habitación de la familia. En Japón, el crédito habitacional se encuentra fuertemente restringido, lo que obliga a las personas a un esfuerzo sistemático y prolongado de ahorro; en el lapso que media hasta que alcanzan el monto exigido, sus fondos quedan disponibles para la inversion.

También contribuye a estimular el espíritu de ahorro el sistema con que las empresas pagan a sus trabajadores, el cual incluye bonos semestrales, de monto considerable, hasta el punto que pueden llegar a representar entre un tercio y un cuarto del salario real. Por su parte, el sistema de pensiones se basa en el pago de una suma importante de una sola vez, otro factor que refuerza la propensión al ahorro. La precariedad del sistema nipón de seguridad social induce a las familias a canalizar recursos significativos para afrontar la vejez o contingencias de salud (McGraw, 1986). Adicionalmente, los depósitos en las cuentas individuales of familiares pueden hacerse en todas las oficinas postales.

La elevada propensión de los nipones al ahorro no obedece entonces, al menos no totalmente, a factores culturales. Ella se ve complementada por mecanismos que tienden a garantizar que ese ahorro se canalice hacia la inversión. El sistema de intermediación financiera, aunque de propiedad privada, está sujeto a regulaciones directas del Banco de Japón y del Ministerio de Finanzas. Ellas obligan a canalizar determinados porcentajes de los recursos disponibles hacia los sectores de particular densidad de capital a los que el pais haya decidido conceder prioridad. Hasta hace poco, el sistema de control limitaba severamente la salida de capitales del país. Es decir, el ahorro quedaba en Japón y se canalizaba, por lo menos en determinadas proporciones, a sectores prioritarios. Esta politica se aplica en el marco de sucesivos programas sectoriales, cuyo objetivo principal consiste en materializar el círculo virtuoso de crecimiento con competitividad.

En la constitución de un sistema industrial altamente competitivo en Japón desempeñaron un papel significativo algunos elementos que están por completo ausentes en la experiencia de los Estados Unidos. Entre ellos destaca el conjunto de disposiciones orientadas a favorecer la adquisición de tecnología en el exterior, mediante la cooperación entre distintas empresas y la coordinación de tales esfuerzos de acuerdo con los criterios de prioridades sectoriales definidos por el мiтr. Pieza clave en esta estrategia es el denominado reverse engineering, que se traduce en la adquisición de bienes tecnológicamente avanzados, con el objeto de desarmarlos, reconstruirlos y mejorarlos en el país.

De otro lado, y a diferencia del resto de los países avanzados y de América Latina, las autoridades niponas adoptaron una política extraordinariamente restrictiva frente a la inversión extranjera en actividades manufactureras, toda vez que consideraban que el mercado interno era la base principal de aprendizaje para la industria nacional. Cederlo a empresas extranjeras significaba poner en grave peligro la posibilidad de que las empresas nacionales adquiriesen el conocimiento e incursionaran posteriormente en los mercados externos. Más aún, la conocida política nipona de control de importaciones favoreció la competencia interna entre las empresas japonesas, aunque en el marco de un mercado cautivo.

Otro aspecto relevante está representado por el componente sectorial de la política fiscal, de antigua data en el caso japonés. Desde la época Meiji, el sector público asume con gran realismo la tarea no sólo de cautelar los equilibrios macroeconómicos, sino de asignar partidas específicas para determinadas áreas -industria naval, ferrocarriles, minería e industria textil de la seda-, a las que se había otorgado prioridad. Se configura así una diferencia fundamental con la concepción de política macroeconómica prevaleciente en el resto de los paises industrializados. En ellos -y sobre todo en los Estados Unidosse sustenta el principio de la neutralidad intersectorial, aduciéndose que las prioridades deben ser definidas por el mercado.

También en el ámbito tributario se manifiesta nítidamente la preferencia que el Estado nipón otorga al sector industrial. Dentro de este último, por otra parte, se privilegian las áreas que exhiben mayor contenido de cambio técnico o potencial impulsor del mercado doméstico o internacional. En 1981 la relación de impuestoventas para el conjunto de las actividades económicas en Japón era levemente más alta que en los 
Estados Unidos (1.9 vs 1.1\%). En este último el coeficiente impuesto-ventas en la industria química y de maquinaria triplica la relación global; en Japón, los coeficientes respectivos son 1.5 y 1.8. A la inversa, en los Estados Unidos el sector financiero soporta un gravamen de $1.4 \%$, mientras que en Japón asciende a $2.3 \%$. Es decir, la tasa tributaria global es levemente superior en Japón, pero bastante menor en las áreas industriales con alto contenido técnico, y más alta en el sector financiero.

En consecuencia, sin detrimento de los factores de índole cultural o religiosa, puede afirmarse que en el prosaico ámbito de la economía operan instrumentos que explican el peculiar perfil de industrialización de Japon, y una cuota apreciable del éxito alcanzado por ese país en el empeño de compatibilizar crecimiento y equidad. La frecuente referencia a Confucio para explicar el "éxito" del Sudeste asiático resulta poco coherente, con el hecho de que hasta hace poco se intentaba explicar el atraso de China aludiendo al mismo personaje.

En lo tocante a la equidad, vale la pena recordar que los mayores avances se lograron durante la ocupación del territorio japonés por parte de fuerzas norteamericanas. En ese período se debilitó el poder de los grandes conglomerados y se llevó a cabo una redistribución de la propiedad agrícola y de las grandes propiedades urbanas (Mizoguchi, 1985).

Es interesante destacar que estas diferencias tan trascendentes a nivel de instrumentos especificos de política económica se dan, no obstante, el hecho de que la importancia cuantitativa del sector público japonés es semejante a la que se observa en Estados Unidos. En ambas economías, la significación del gasto público y el papel que las empresas públicas desempeñan en la producción industrial son más moderados que en el conjunto de paises industrializados europeos y, particularmente, que en Alemania Federal. Esta aparente similitud institucional encubre diferencias fundamentales de orientación en el uso de los instrumentos del sector público. Más aún, el patrón japonés de industrialización presenta numerosos elementos en común con el de Alemania Federal. Sin embargo, el peso relativo del sector público, tanto en el producto bruto como en las empresas del sector índustrial, es notablemente superior en el caso germano.

Las magnitudes agregadas constituyen, pues, un dato muy insuficiente cuando se quiere avanzar en la comprensión del papel del Estado en el proceso de industrialización de un país. $\mathrm{La}$ vinculación casi simbólica que se observa entre el Estado y los grandes grupos empresariales en Japón hace innecesaria una mayor presencia directa del sector público en las actividades productivas. La baja significación cuantitativa del Estado nipón tiene poco que ver con el fenómeno, a primera vista semejante, que se observa en la economía estadounidense, toda vez que en ella la articulación del sector público con el privado es prácticamente nula (Lodge y Vogel, 1987). A la inversa, la relación entre el sector público, la intermediación financiera y el sector industrial en Alemania Federal se asemeja mucho a la japonesa, pese a que en el gigante asiático la gravitación del Estado en la economía es sustancialmente menor (Zysman, 1984).

Tanto en los Estados Unidos como en Japón el déficit público representa en los últimos años aproximadamente $5 \%$ del producto. Pero mientras en el primero el déficit equivale al monto total de ahorros privados disponibles, en el caso japonés la relación es de apenas $35 \%$. La gravitación del sector público y la importancia relativa de su déficit son entonces semejantes, pero sus efectos, muy distintos (McGraw, 1986).

\section{IV \\ Competitividad y políticas postkeynesianas}

De lo expuesto se desprende que en el mercado internacional compiten no sólo empresas. Se confrontan también sistemas productivos, esque- mas institucionales y organismos sociales, en los que la empresa constituye un elemento importante, pero integrado en una red de vinculacio- 
nes con el sistema educativo, la infraestructura tecnológica, las relaciones gerencial-laborales, el aparato institucional público y privado, el sistema financiero, etcétera.

En las naciones industrializadas el debate en torno al mejoramiento de la competitividad se da en el marco de instituciones cuya legitimidad nadie cuestiona. De otra parte, la cohesión social es bastante fuerte. Los patrones de consumo y el acervo de conocimientos tecnológicos se han difundido y homogeneizado. La inserción internacional tiene como eje al sector manufacturero.

En pos del objetivo de acrecentar la competitividad, los gobiernos de estos países impulsan programas de apoyo a los sectores de alto contenido tecnológico, readecuan y vigorizan el sistema educativo y de investigación y desarrollo, llevan a cabo programas preferenciales de apoyo a la pequeña y mediana industria, crean condiciones favorables a la cooperación entre empresas y de éstas con el sector público, promueven la reforma del sistema de relaciones industriales y estudian programas públicos de inversión para el mejoramiento de la infraestructura, con énfasis en las telecomunicaciones. Las empresas, por su parte, exploran nuevas formas de organización y de vinculación con el sector académico y con capitales de riesgo, y ponen a prueba diversas modalidades de relaciones industriales, con el propósito de estimular la cooperación y motivación de los trabajadores; y desarrollan las más variadas modalidades de cooperación entre ellas, con los gobiernos y agrupaciones regionales, especialmente en el ámbito de la investigación y el desarrollo.

Estaríamos asistiendo pues a la generación de estrategias susceptibles de ser calificadas de postkeynesianas (Freeman, 1987). El fenómeno, que está lejos de haberse decantado, coexiste con la vigencia de una retórica y de una praxis de agresivo carácter prekeynesiano. La institucionalidad de los países avanzados - que asegura a los distintos actores sociales y políticos el derecho a participar activamente en la defensa de sus posiciones-impide que las políticas prekeynesianas inhiban el surgimiento de estas otras.

A la luz de nuestro análisis, lo más probable, incluso, es que la estrategia postkeynesiana configure el marco en que se desarrolle el quehacer económico en las próximas décadas.

\section{Bibliografia}

Antonelli, C. (1987): Les nouvelles technologies de linformation et l'organisation industrielle. Experiences et lendances en Italie. OCDE, julio.

Arnold, E. y G. Ken (1987): Information technology and emerging growth areas. Policy option for promating growth trough information technology. Science Policy Research Unit, University of Sussex.

BIs (Banco de Pagos Internacionales) (1986): Fifty-sixth annual report, Basilea.

Boyer, R. (1983a): Economic policy: past and present. (1983b): L'introduction du taylorisme en France. Centre d'Etudes Prospectives d'Economie Mathematique Apliquees a la Planification, cepremap, No 8313. Paris.

Boyer, R. y I. Mistral (1983): La crise actuelle: d'une analyse historique a une vue prospective. CEPREMAP, $\mathrm{N}^{\circ} 8304$.

Branson, W. y otros (1980): Trends in United States international trade and investment since World War II. $M$. Feldstein (comp.), The American economy in transition, University of Chicago Press.

Brown, C. y H. Bennett (1986): Industrial restructuring and labor relations. Industrial relations, Vol. 25, $\mathrm{N}^{\circ} 2$.

Daudestadt, L. (1987): Free markets versus political consensus. The international competitiveness of societies. Intereconomics, mayoljunio.

Denison, E. (1980): The contribution of capital to economic growth. American Economic Assaciation, mayo.
Drucker, P. (1987a): Japan choices, Foretgn affairs, Vol. 65, $\mathrm{N}^{\mathrm{D}} 5$.

(1987b): The trasnational economy. The Wall Street Journal. 25, agosto.

Freeman, C. y L. Soete (1982): Unemployment and technical innovation: a study of long toves and economic development. Frances Pinter, Londres.

(1985): Information, technology and employment; an assessment. Science Policy Research Unit, University of Sussex.

Freeman, C. (1987): The future of information and communication technologies: implications for decision-makers. Science Policy Research Unit, University of Sussex, february.

Ladge, G. (1986): The new American ideology. New York University Press, Washington Square, Nueva York.

Lodge, G. y E. Vagel, eds. (1987): Ideology and national competitiveness. An analysis of nine countries. Harvard Business School, Baston, Massachusetts.

McCormick, J. (1987): France: ideological divisions and the global reality. G. Lodge y E. Vogel (comps.).

McGraw, T. (1986): America versus Japan. A comparative study. Harvard Business School.

Mensh, G. (1979): Sealemate in technology: innotations overcome the Depression. Ballinger, Cambridge, Massachusets.

Messine, Ph. (1984): Les saturniens. Quiand les patrons reinventent la société. Piore y Sabel (comps.). 
(1985): Liberté, egalité, modernite. Promesses at menaces de la modernisation. Editions La Decouverte, Paris.

Mizoguchi, T. (1985): Economic development policy and income distribution: the experience in East and South. east Asia. The developing economies, diciembre.

Oxford Analytica (1986); America in perspective. Houghton Mifflin Co., Boston.

Pfaller, A. (1987): Are the Western welfare states still competitive? Intereconomics, mayo/junio,

Piore, M. y Ch. Sabel (1983): Italian small business development: lessons for US industrial policy. Zysman, J. y L. Tyson (comps.).

(comps.) (1984): The second industrial divide.

Piore, M. (1986): Perspectives on labor market flexibility Industrial relations, Vol, $25, \mathrm{~N}^{\circ} 2$.
Ribeiro, J. y otros (1987): Trois economies sud-europennes dans la CEE a douze: Italie, l'Espagne et le Portugal. Economie prospective istlemationale.

Rosenberg, N. y C. Frischtak (1984): Technological innovation and long waves. Cambridge Journal of Economics.

Schumpeter, ]. (1950): Capitatism, socialism and democracy. George Allen and Unwin, Londres.

Zysman, J. y L. Tyson (1983): American industry in intemational competition. Cornell University Press, Itaca y Londres.

Zysman, J. (1984): Governments, markets and grouth. Financial systems and the politics of ind tustrial change. Cornell University Press, Itaca y Londres. 